\title{
Band-Reject Filters for Bragg Mirror Using Perturbed Chiral Sculptured Thin Films
}

\author{
Zahir Muhammad ${ }^{1}$, Mukhtar Ahmad ${ }^{2}$ and Li Song ${ }^{3}$ \\ 1. National Synchrotron Radiation Laboratory \& University of Science and Technology of China, Hefei, Anhui 230029, China \\ 2. Department of Physics, Islamia College University, Peshawar 25120, Pakistan \\ 3. National Synchrotron Radiation Laboratory \& University of Science and Technology of China, Hefei, Anhui 230029, China
}

\begin{abstract}
We have theoretically simulated the optical properties of perturbed chiral STF (sculptured thin film) with and without defect. It was revealed that such STFs could act as band-reject filters with tunable Bragg regime of free space wavelengths and different bandwidth through changing the vapor incident angle during glancing angle deposition process. These Bragg filters are narrowing due to the change of vapor incident angle for circularly polarized light. Interestingly, as the vapor incident angle increased, the band-rejected filter exhibited to decrease the bandwidth and shifted in the Bragg regime, indicating that such STF nanostructures have high potential for behaving as unique polarization insensitive Bragg mirror.
\end{abstract}

Key words: Bragg phenomenon, sculptured thin films, optical filters, nano-helixes, Bragg mirrors.

\section{Introduction}

Sculptured thin films of helicoidally shaped morphologies are called CSTFs (chiral sculptured thin films) [1]. These shapes are fabricated, most commonly, using physical vapor deposition by evaporating and directing the vapors from a source towards a rotating substrate tilted at an angle $\chi_{v}$ experimentally. The local tilt or column angle $\chi$ growing on the substrate as a function of vapor incident angle $\chi_{v}$. Usually chiral STFs (sculptured thin films) consist of helical nanowires oriented perpendicular to the surface of any substrate. Structurally chiral material, as well as molecules exhibits helical orientation order $[2,3]$. Structurally chiral materials fabricated as a stacks using thin film technology $[1,4]$. Chiral STFs have the property that reflects circularly polarized light of one handedness, but a very little of the other, in the Bragg regime of free space wavelength which is called circular Bragg phenomenon and the Bragg regime is called circular Bragg regime [5]. Structurally chiral

Corresponding author: Zahir Muhammad, Ph.D., research student, research fields: nanophotonics and nanomaterials, email: zmuhammad@student.qau.edu.pk. materials have the property of circular Bragg phenomenon [1]. Chiral STFs of multi-section structures produced a spectral hole in the Bragg regime which contained either chiral STFs layer or twist defects or both in the middle [6-8]. The selective remittances for circular polarization of light have the CP-dimorphic optical response, property can be exploited for a variety of optical filters and sensors [7] using matched chiral STFs. Due to porosity, the chiral STFs are used for optical sensing in humidity and various chemicals [6, 10, 11]. Circular Bragg phenomenon in chiral STFs has the property which is used as circular polarization filters [12], which may be polarization sensitive or insensitive. Circular polarization insensitive band rejection or bandpass filters are fabricating using cascaded chiral STFs $[13,1]$. The phase discontinuity in the center of the periodic structure introduces a hole in the Bragg regime of circular Bragg phenomenon which is acting as filters. The spectral hole appears in the Bragg gratings [14] and narrow bandpass filtering in optical fiber communication [8], with increasing the thickness of the chiral STF. The spectral hole in the Bragg regime 
produced narrow passband which is die out and a new ultranarrow spectrum is occurring in the Bragg regime of free space wavelength due to increasing thickness [15]. Multiple spectral holes are fabricated in the Bragg regime by inserting twist defects which are different from each other $[15,16]$. Same as the tilt-modulated chiral STF having the property of circular Bragg phenomenon and spectral hole designing [15]. These sculptured thin films are porous and its porosity is inversely proportional to the refractive index of the materials. We use GLAD (glancing angle deposition) technology which fabricates optical thin films, by controlling the refractive index and porosity [17]. Here, in this manuscript we see the optical response of perturbed chiral sculptured thin films fabricated by using GLAD process to find out the following issues. We are studying that how the spectrum of the circularly polarized light is controlled over vapor incident angle using perturbed chiral STFs and how the chiral STFs changes the tolerance of the Bragg regime is controlled to fabricate different optical filters in the direction of vapor flux using GLAD process? How without and as well as defect STFs are used for the fabrication of Bragg mirror and band-reject filters in different regimes of wavelength?

The mathematical description for the chiral STFs is presented and described in sec. 2. The numerical discussion is presented in sec. 3 and concluding remarks are in sec. 4. In exp ${ }^{(i \omega t)}$ time, dependency is implicit, with which $\omega$ is the angular frequency and $t$ is the time. Whereas, the $k_{o}=\omega \sqrt{\varepsilon_{\mathrm{o}} \mu_{\mathrm{o}}}, \lambda_{o}=2 \pi / k_{o}$, denoted the wavelength and wave-number while $\eta_{o}=\omega \sqrt{\mu_{\mathrm{o}} / \varepsilon_{0}}$, the intrinsic impedance of free space respectively. Here $\varepsilon_{0}$ and $\mu_{\mathrm{o}}$ are the perm-itivity and permeability of free space corresp-onddingly. The vectors are denoted by boldface letters and for dyadics we have used twice underlined. The Cartesian unit vectors are represented as $\mathbf{u}_{\mathbf{x}}, \mathbf{u}_{\mathbf{y}}$, and $\mathbf{u}_{\mathbf{z}}$.

\section{Theory}

Let's consider the schematics diagram of the chiral STFs without as well as defect at $\mathrm{z}=0$ structures is shown in Fig. 1.

These chiral STFs are periodically nonhomogeneous. Therefore the permittivity of these STFs is written in the dyadic form as:

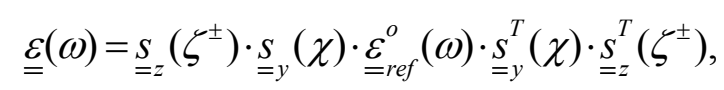

where the rotation dyadic of the STFs is written as:

$$
\begin{gathered}
\underset{=s}{S}\left(\zeta^{ \pm}\right)= \\
-\mathrm{u}_{y} \mathrm{u}_{y}+\left(\mathrm{u}_{x} \mathrm{u}_{x}+\mathrm{u}_{z} \mathrm{u}_{z}\right) \cos \left(\zeta^{ \pm}\right)+\left(\mathrm{u}_{z} \mathrm{u}_{x}\right. \\
\left.-\mathrm{u}_{x} \mathrm{u}_{z}\right) \sin \left(\zeta^{ \pm}\right),
\end{gathered}
$$

and shape dyadic of chiral STF is,

$$
\begin{gathered}
S_{y}(\chi)=\mathrm{u}_{y} \mathrm{u}_{y}+\left(\mathrm{u}_{x} \mathrm{u}_{x}+\mathrm{u}_{z} \mathrm{u}_{z}\right) \cos \chi+\left(\mathrm{u}_{z} \mathrm{u}_{x}-\right. \\
\left.\mathrm{u}_{x} \mathrm{u}_{z}\right) \sin \chi .
\end{gathered}
$$
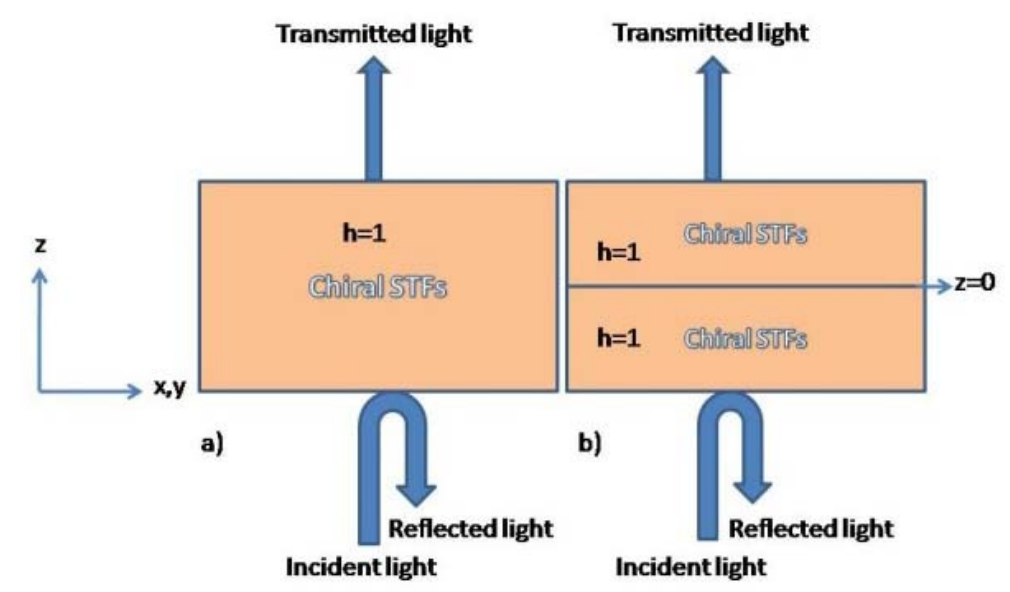

Fig. 1 Schematics of chiral STF having defect and without defect. 
From Eq. (1), the relative permittivity dyadic $\varepsilon_{\text {ref }}^{o}(\omega)$ of the local orthorhombic symmetry of the STF is expressed through a dyadic function as:

$$
\varepsilon_{r e f}^{o}(\omega)=\varepsilon_{a}(z) \mathrm{u}_{z} \mathrm{u}_{z}+\varepsilon_{b}(z) \mathrm{u}_{x} \mathrm{u}_{x}+\varepsilon_{c}(z) \mathrm{u}_{y} \mathrm{u}_{y}
$$

where $\varepsilon_{a, b, c}(z)$ is scalar relative permittivity of the STF. In Eq. (1), $\chi$ is the tilt angle which varies as a function of depth into the film (along the $z$-axis) which is further as a function of vapor incidence angle $\chi_{v}$. In Fig. $1, \chi$ is the tilt relative to the $x y$ plane. The relation of column angle $\chi$ and deposition angle $\chi_{v}$ without modulation using Tait's rule, which measures the porosity and index of refraction using tangent rule for the GLAD process [18] as:

$$
\chi=\chi_{v}+\sin ^{-1}\left(\frac{1-\cos \chi_{v}}{2}\right) .
$$

The defect structures of chiral STFs from Eq. (1), we can write as:

$$
\zeta^{ \pm}=h\left(\frac{\pi z}{\Omega}+\xi^{ \pm}\right)
$$

where $2 \Omega$ is the structural period and $h$ is structural handedness which can be either +1 or -1 to indicate one of the two types of structural handedness. The structural period of the CSTF and $\xi^{+}-\xi$ represents the defect at $z=0$.

To study the optical response of these chiral STFs we considered incident plane waves of circular polarization states excited on the CSTF nanostructures that have been formulated elsewhere [1]. After using boundary-value problem to find the reflectances and transmittances whereas, the chiral STF has been modeled using piecewise-uniform approximation [1]. The chiral STF is divided into thin slices parallel to the $x y$ plane in this technique and the permittivity of the chiral STF is taken to be uniform which is same as of the middle slice. In this technique thickness of slice is taken $0.5 \mathrm{~nm}$ after ascertaining that reflectances and transmittances converged within $0.5 \%$ of their value when the slice thickness was $5 \mathrm{~nm}$ and $0.1 \%$ when it is $1 \mathrm{~nm}$.

\section{Numerical Results and Discussion}

For numerical results, we considered the principle relative permittivities $\varepsilon_{a, b, c}$ of the perturbed columnar thin film of titanium oxide ( $\mathrm{TiO} 2)$. This is modulated. The relationship between $\chi$ and $\chi_{v}$ with $\varepsilon_{a, b, c}$ has been measured by Hodgkinson et al. [19], for the experimental data titanium oxide as follow,

$$
\begin{aligned}
& \varepsilon_{a}=\left[1.0443+2.7394\left(\frac{\chi_{\mathrm{v}}}{\pi / 2}\right)-1.3697\left(\frac{\chi_{\mathrm{v}}}{\pi / 2}\right)^{2}\right]^{2} \\
& \left.\varepsilon_{b}=\left[1.6765+1.5649\left(\frac{\chi_{\mathrm{v}}}{\pi / 2}\right)-0.7825\left(\frac{\chi_{\mathrm{v}}}{\pi / 2}\right)^{2}\right]^{2}\right\}, \\
& \left.\varepsilon_{c}=\left[1.3586+2.1109\left(\frac{\chi_{\mathrm{v}}}{\pi / 2}\right)-1.0554\left(\frac{\chi_{\mathrm{v}}}{\pi / 2}\right)^{2}\right]^{2}\right]
\end{aligned}
$$

where,

$$
\chi_{v}(z)=\tan ^{-1}\left(\frac{\tan \chi(z)}{2.8818}\right),
$$

Furthermore, we chose $\Omega=200 \mathrm{~nm}, \mathrm{~h}= \pm 1$, and $\chi_{v}$ was kept variable.

The remittance spectrum of circularly polarized light of unperturbed chiral STF without defect as a function of free-space wavelength is shown in the Fig. 2. Fig. 2 shows that light is maximally reflected in the Bragg regime which behaves as a perfect reflector. The transmittance spectrum $T_{R R}$ shows that the unperturbed chiral STF behaves as Bragg mirror in the Bragg regime. Similarly for perturbed chiral STF without defect using GALD process we observed the same effect but, the bandwidth is decreasing and its center wavelength is shifting in the Bragg regime as we increase the vapor incident angle. Fig. 3 shows that Bragg regime is shifted but, the phenomenon is still occurring in CSTF without defect on perturbation. The band of perfect reflector and band-reject filters is decreasing due to perturbation. Even further increasing the value of $\chi_{v}$ the given phenomenon is shifted further in the Bragg regime and its bandwidth is more decreasing which are seen from Fig. 4. The transmittance is acting as band-reject filters. Moreover, by further increasing the vapor incident angle through GALD process the given phenomenon is disappearing 

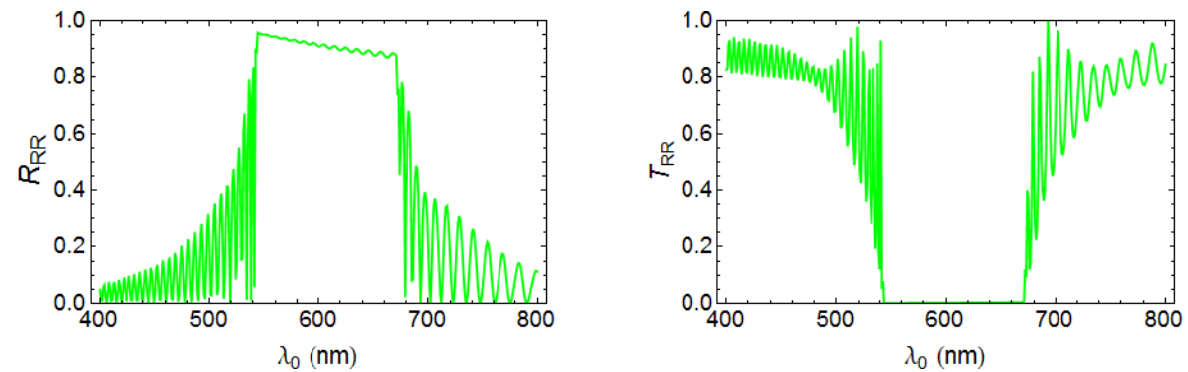

Fig. 2 Remittance spectrum as a function of $\lambda$ of $h= \pm 1, \chi_{v}=0^{\circ}, \Omega=200 \mathrm{~nm}, L=60 \Omega$ and normal incidence.
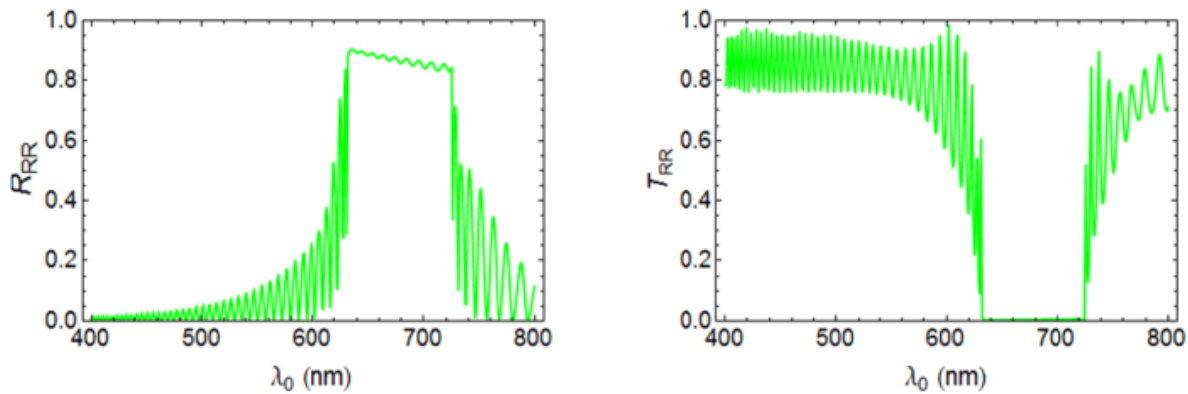

Fig. 3 Same as Fig. 2, except $\chi_{v}=10^{\circ}$.
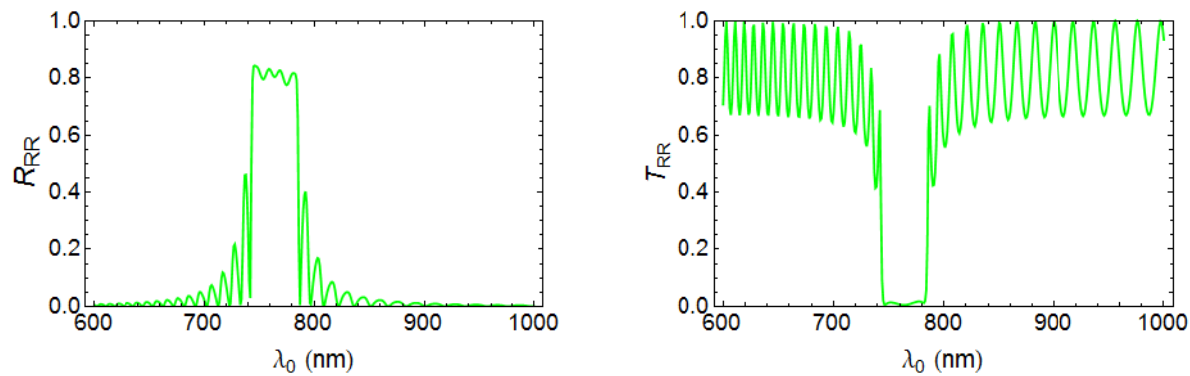

Fig. 4 Same as Fig. 2, except $\chi_{v}=25^{\circ}$.

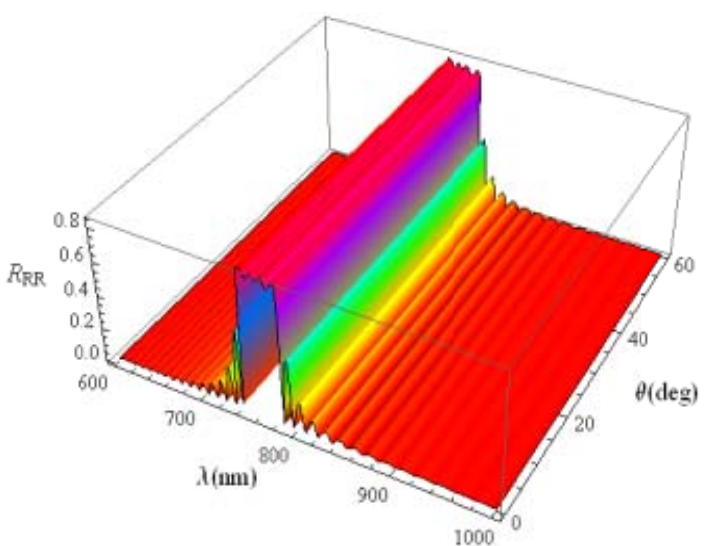

Fig. 5 Same as Fig. 4, except varying incident angle.

in that Bragg regime and shifted in another regime and will narrower even; it will disappear in the Bragg regime. In Fig. 5, we examined the effect of incident angle on the remittance spectrum using without defect perturbed chiral STFs. We examined that, oblique

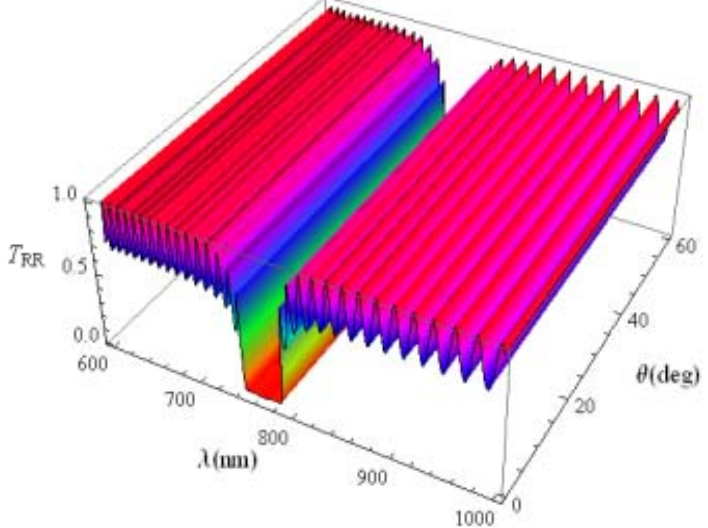

incident angle cannot affect the same phenomenon in the Bragg regime. We can see that, for a same vapor incident angle perfect reflector and band-reject filter is occurring for each angle in the same Bragg regime and center wavelength. 

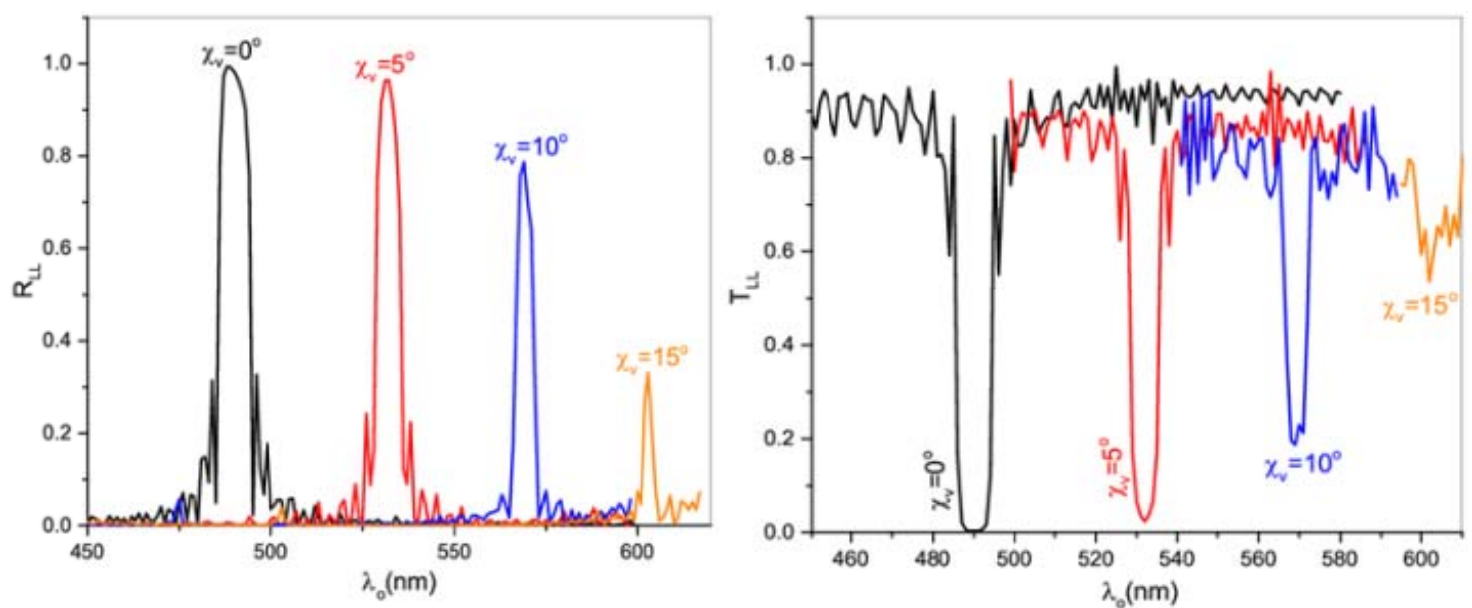

Fig. 6 Remittances spectrum of a normally excited as a function of $\lambda$ of $h= \pm 1, \Omega=200 \mathrm{~nm}, L=30 \Omega$ and $\chi_{\mathrm{v}}=\left\{0^{\circ}, 5^{\circ}, 10^{\circ}, 15^{\circ}\right\}$.
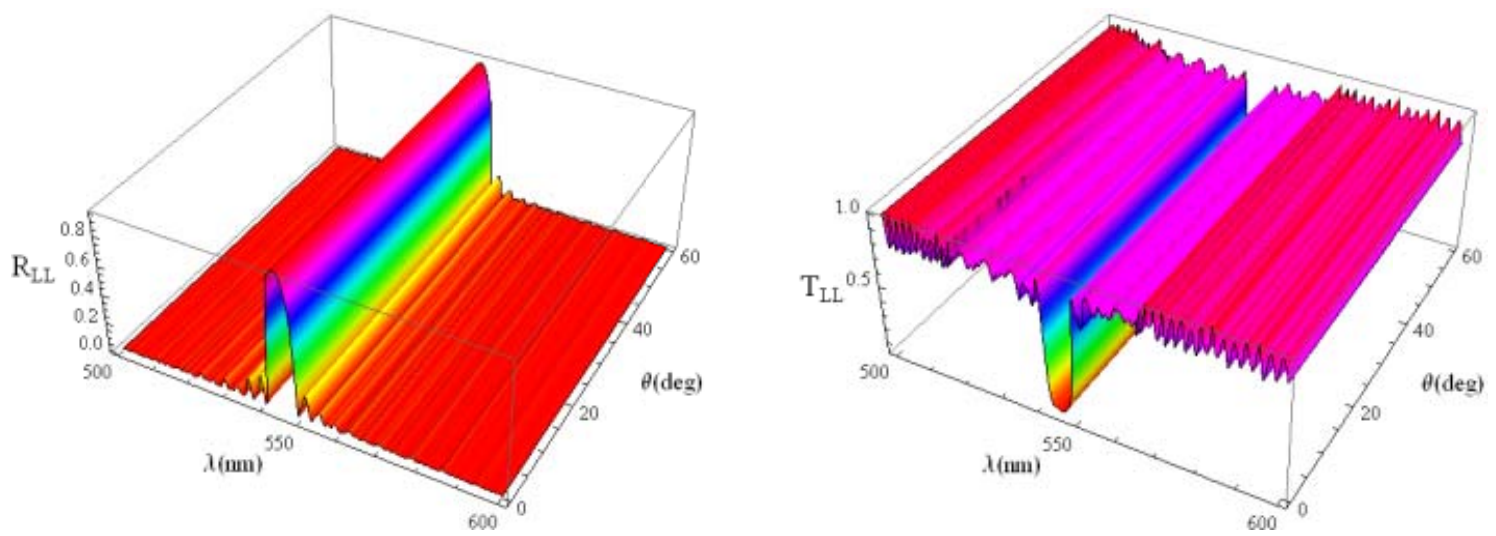

Fig. 7 Same as Fig. 6, except $\chi_{v}=10^{\circ}$ and varying incident angle.

Furthermore let's use defect nanostructures of perturbed chiral STF as shown in the Fig. $1 \mathrm{~b}$ to observe its optical properties. The computed remittance spectrums for LCP (lift circularly polarized) incident light is represented by $R_{L L}$ and $T_{L L}$ which are computed with a wavelength resolution of $0.1 \mathrm{~nm}$ as shown in the Fig. 6 . The transmittance spectrum $T_{L L}$ from the perturbed defect chiral STFs shows band-reject filters and reflectance $R_{L L}$ shows Bragg mirror. When $\chi_{\mathrm{v}}$ increases from $0^{\circ}$, to higher value the chiral STF still behaves as band-reject filters for same circular polarization states of incident light. However, though increasing values of $\chi_{\mathrm{v}}$ the bandwidth and the central frequency are different from each other in the Bragg regime. Moreover, further increasing the vapor incident angle the given phenomenon will be disappeared and it will be narrower and it will shift to higher regime of free-space wavelength. The same were observed for different incident angles. From Fig. 7, it is seen that, the incident angle cannot affect the circular Bragg phenomenon which is fabricating band-reject filters. Therefore, for different incident angles of same $\chi_{\mathrm{v}}$, it is viewed that, the filters have bandwidth and center wavelength.

\section{Concluding Remarks}

We conclude that, in the Bragg regime we find band-reject filters when a uniform band gap is sandwiched between two identical perturbed chiral STFs or central defect in the perturbed CSTF using circularly polarized light. In the Bragg regime we have observed that the perturbed chiral STF permits light of particular polarization state in different regimes using different vapor incident angles. In these 
Bragg regimes we revealed that it totally reflects light and no transmission which, fabricated band-reject filters and perfect reflectors. The circular Bragg phenomenon shown here as a wide band-reject filters and polarization insensitive Bragg mirrors for chiral STF with defect. But, it is attained that, different band-reject filters have different bandwidth and central frequency using different vapor incident angles. Therefore polarization insensitive sensitive band-reject filters and perfect reflectors make polarization insensitive Bragg mirrors for circularly polarized light using perturbed chiral STFs.

\section{Acknowledgement}

The financial support was from the National Basic Research Program of China (2014CB848-900), the National Natural Science Foundatio of China (U1232131, U1532112, 11375198, 11-574280) and User with Potential from CAS Hefei Science Center (2015HSC-UP020). Z. Muhammad acknowledges the CSC fellowship for financial support.

\section{References}

[1] Lakhtakia A., and Messier, R. 2005. Sculptured Thin Films Nanoengineered Morphology and Optics. SPIE Press.

[2] Li, Q. 2014. Nanoscience with Liquid Crystals. Springer.

[3] Robbie, K., Broer, D. J., and Brett, M. J. 1999. "Chiral Nematic Order in Liquid Crystals Imposed by an Engineered Inorganic Nanostructure.” Nature 399 (6738): 764-6.

[4] Miles, M. W., Brian, J. G., and Clarence, C. 2007. "Thin Film Precursor Stack for MEMS Manufacturing." U.S. Patent 221 (7): 495.

[5] Muhammad, Z., Faryad, M., and Naqvi, Q. A. 2014. "Suppression of Circular Bragg Phenomenon in Tilt Modulated Chiral Sculptured Thin Films at Oblique Incidence." Opt. Eng. 53 (11): 117110.

[6] Wang, F., and Lakhtakia, A. 2003. "Specular and Nonspecular, Thickness-Dependent, Spectral Holes in a Slanted Chiral Sculptured Thin Film with a Central Twist
Defect.” Optics Commun. 215 (1): 79-92.

[7] Wang, F., and Lakhtakia, A. 2005. "Optical Crossover Phenomenon due to a Central 90-Twist Defect in a Chiral Sculptured Thin Film or Chiral Liquid Crystal." In Proceedings of the Royal Society of London A: Mathematical, Physical and Engineering Sciences 461 (2061): 2985-3004.

[8] Lakhtakia, A., and McCall, M. 1999. "Sculptured Thin Films as Ultranarrow Bandpass Circular Polarization Filters." Optics Commun. 168 (5): 457-65.

[9] Lakhtakia, A. 2004. Nanometer Structures: Theory, Modeling, and Simulation. SPIE Press.

[10] Liu, Y. J., Shi, J., Zhang, F., Liang, H., Xu, J., Lakhtakia, A., Fonash, S. J., and Huang, T. J. 2011. "High-Speed Optical Humidity Sensors Based on Chiral Sculptured Thin Films." Sens. \& Actuat. B: Chem. 156 (2): 593-8.

[11] Zourob, M., and Lakhtakia, A. 2010. Optical Guided-Wave Chemical and Biosensors II. Vol. 8. Springer Science \& Business Media.

[12] Wu, Q., Hodgkinson, I. J., and Lakhtakia, A. 2000. "Circular Polarization Filters Made of Chiral Sculptured Thin Films: Experimental and Simulation Results." Opt. Eng. 39 (7): 1863-80.

[13] Suzuki, M., and Taga, Y. 2001. "Integrated Sculptured Thin Films." Jap. J. of App. Phy. 40 (4A): L358.

[14] Torres, P., and Valente, L. G. 2002. "Spectral Response of Locally Pressed Fiber Bragg Grating." Optics Commun. 208 (4): 285-91.

[15] Muhammad, Z., Naqvi, Q. A., and Faryad, M. 2015. "Spectral Hole Filters Using Tilt-Modulated Chiral Sculptured Thin Films.” Opt. Commun. 346: 178-82.

[16] Lakhtakia, A. 2007. "Generation of Spectral Holes by Inserting Central Structurally Chiral Layer Defects in Periodic Structurally Chiral Materials." Optics Commun. 275 (2): 283-7.

[17] Jen, Y. J., and Lin, C. F. 2008. "Anisotropic Optical Thin Films Finely Sculptured by Substrate Sweep Technology." Opt. Exp. 16 (8): 5372-78.

[18] Tait, R. N., Smy, T., and Brett, M. J. 1993. "Modeling and Characterization of Columnar Growth in Evaporated Films." Thin Solid Films 226 (2): 196-201.

[19] Hodgkinson, I. J., Wu, Q. H., and Hazel, J. 1998. "Empirical Equations for the Principal Refractive Indices and Column Angle of Obliquely Deposited Films of Tantalum Oxide, Titanium Oxide, and Zirconium Oxide." Appl. Opt. 37 (13): 2653-9. 